\title{
All-organic and organic-silicon photonic ring micro-resonators
}

\section{Antao Chen, Larry Dalton, Travis Sherwood, Alex K.-Y. Jen, Payam Rabiei, et al.}

Antao Chen, Larry Dalton, Travis Sherwood, Alex K.-Y. Jen, Payam Rabiei, William Steier, Yanyi Huang, George T. Paloczi, Joyce K. S. Poon, Axel Scherer, Amnon Yariv, "All-organic and organic-silicon photonic ring microresonators," Proc. SPIE 5708, Laser Resonators and Beam Control VIII, (12 April 2005); doi: 10.1117/12.609082

Event: Lasers and Applications in Science and Engineering, 2005, San Jose, California, United States 


\title{
All-organic and organic-silicon photonic ring micro-resonators
}

\author{
Antao Chen $^{\text {a,b,* }}$, Larry Dalton ${ }^{\text {c,b }}$, Travis Sherwood ${ }^{\mathrm{d}}$, and Alex K.-Y. Jen ${ }^{\mathrm{d}}$ \\ Applied Physics Laboratory ${ }^{a}$, Department of Electrical Engineering ${ }^{\mathrm{b}}$, Department of Chemistry ${ }^{\mathrm{c}}$, \\ and Department of Material Science and Engineering ${ }^{\mathrm{d}}$ \\ University of Washington, Seattle, WA 98195 \\ Payam Rabiei and William Steier \\ Department of Electrical Engineering, University of Southern California, Los Angeles, CA 90089 \\ Yanyi Huang, George T. Paloczi, Joyce K. S. Poon, Axel Scherer, and Amnon Yariv \\ Department of Applied Physics, California Institute of Technology, Pasadena, CA 91125
}

\begin{abstract}
Organic electro-optic materials offer exceptional processability (both from solution and the gas phase) that permit fabrication of flexible and conformal device structures and the integration of organic materials with a wide range of disparate materials. In addition, organic electro-optical materials have fundamental response times that are in the terahertz region, and useable electro-optic coefficients that are approaching $300 \mathrm{pm} / \mathrm{V}$ (at telecommunication wavelengths). In addition to fabrication by traditional lithographic methods, multiple devices on a single wafer have been fabricated by soft and nano-imprint lithography. In this presentation, we review the fabrication and performance evaluation of a number of all-organic and organic-silicon photonic ring microresonator devices. Both electrical and thermal tuning of devices, including both single and multiple ring micro-resonators, are demonstrated.
\end{abstract}

Keywords: Optical resonators, optical polymers, integrated optics, two-photon polymerization

\section{INTRODUCTION}

Micro-ring resonators offer significant advantages over the existing telecommunication filter technology and may be the foundation of future dense-wavelength-division-multiplexing (DWDM) filters, modulators, and switches ${ }^{1-5}$. They can also be a new technology platform of miniature optical sensors of temperature, strain/stress, chemical and biological agents ${ }^{6}$. Conventional integrated optics based on Mach-Zehnder interferometers, directional couplers, Ybranch switches, and multi-mode interference (MMI) technologies requires device lengths on the order of a centimeter. In contrast, micro-ring resonator devices have much smaller (several micron to a few hundred micron) sizes. This unique advantage makes them ideal for large scale integration for single chip optical switch arrays and wavelength-division multiplexing modules. It also makes them highly suitable for monolithic vertical integration on silicon integrated circuit chips.

Micro-ring resonators have been demonstrated in many materials, including GaAs and InP, silicon, glass, and polymers. As artificial materials, polymers can have tailored optical (index of refraction, birefringence, transparent window), processability, and tuning/sensor (electro-optic, thermo-optic, photoelastic) properties. Many fabrication options exist for polymer micro-ring resonators. They can be fabricated by photo-patterning, etching, imprinting, softlithography molding, two-photon polymerization, and e-beam writing. They can also be fabricated on flexible, curved, and conformal substrates. These advantages allow polymer micro-ring resonators to have designs, functionality, and performance not available by other materials.

* antaochen@apl.washington.edu; phone 1206 543-1274; photonics.apl.washington.edu 
The theory of basic ring resonators can be found in many references ${ }^{7,8}$. The two most important figures of merit of a resonator are the free spectrum range (FSR), and the Q-value. The free spectrum range is the separation between resonant wavelengths. The Q-value is a measure of the sharpness of the resonance. It is defined as $\lambda / \Delta \lambda$, where $\lambda$ is the resonating wavelength and $\Delta \lambda$ is the width of the resonance. The Q depends on the overall loss of the ring resonator, including the material absorption, scattering, and bending losses. Different applications have different emphasis on the performance. Fiber optic telecom applications want only one resonance inside a wavelength band (1510-1565 for C-band and 1565-1620 for L-band). This means the FSR needs to be as large as $60 \mathrm{~nm}$. The resonance needs to be broad enough to cover the channel bandwidth, which puts an upper limit on the Q-value. On the other hand, sensor applications require a high Q-value because the sensitivity is directly related to the Q-value, and FSR is usually not a concern.

The rest of the paper will discuss about ring resonators made by various technologies, dynamic tuning and permanent trimming, and various devices made with ring resonator structures.

\section{FABRICATION TECHNOLOGIES OF POLYMER RING RESONATORS}

\subsection{Conventional photolithography and etching ${ }^{8}$}

Waveguide micro ring resonators can be made by conventional photolithography and etching. However, photolithography lacks the resolution to define the sub-micro gap between the bus waveguide and the ring waveguide. Therefore it is necessary to fabricate ring and the bus waveguide in different polymer layers, and to vertically couple them, as shown in Figs. 1 and 2. The typical vertical separation between the ring and the bus is 0.5 to $1 \mathrm{um}$. Because it is relatively easy to precisely control (by spin speed) and adjust (by etching) the layer thickness, devices of vertical coupling design have high yields and more consistent performance. It also has a relatively large tolerance to the lateral misalignment between the ring and the bus.
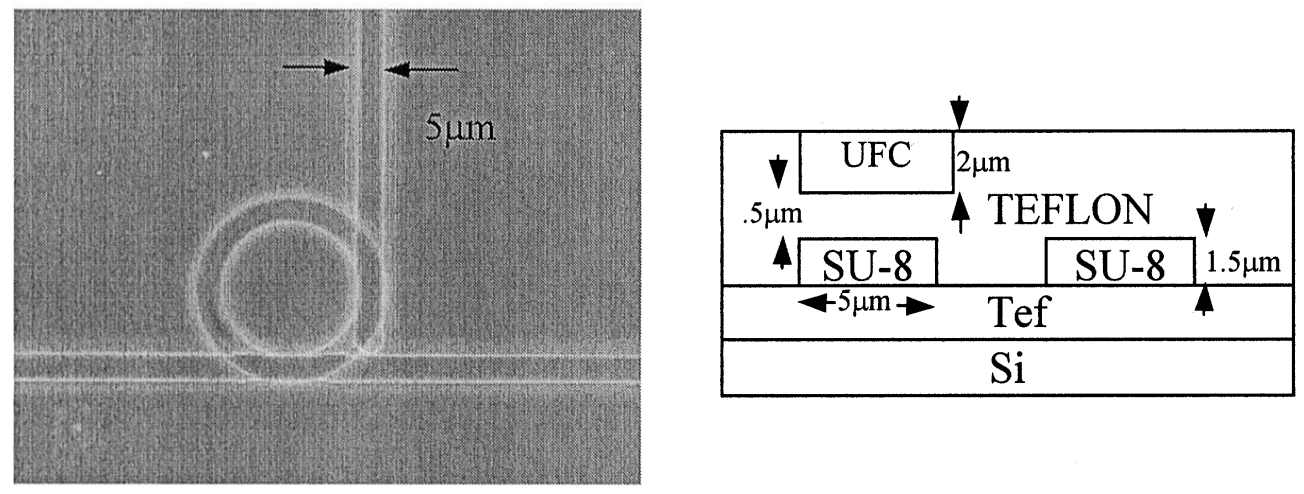

Figure 1: Vertically coupled polymer ring resonator.

\subsection{Soft lithography replication of polymer ring resonators ${ }^{7}$}

Although micro-ring resonators can be made with vertical coupling design and conventional photolithography and etching, there are some limitations. Two lithography and two etching steps are needed, and the substrate has to be a flat wafer for photolithography. An alternative fabrication technique that takes advantage of the plastic nature of EO polymers is soft replica molding lithography ${ }^{9}$. Soft lithography can replicate micro-ring resonator devices in a single step. In the soft lithography process, a master is generated using e-beam patterning of a photoresist. Elastomeric (typically poly(di-methylsiloxane) (PDMS)) stamps or molds are replicated from the master, and the stamps are used to mold polymer waveguide devices. Each master can fabricate many stamps, and each stamp can make many device imprints. Therefore, a large number of devices can be produced easily. Materials that are suitable for this method can 
be either photo-curable, due to the transparency of the stamp material to ultraviolet light, or solvent based polymer solutions, made possible by the permeability of the stamp material to common polymer solvents that allows solvents to evaporate. Because the mold is made of flexible elastomer material, replication can be done on flat, curved, or flexible and conformal substrates.

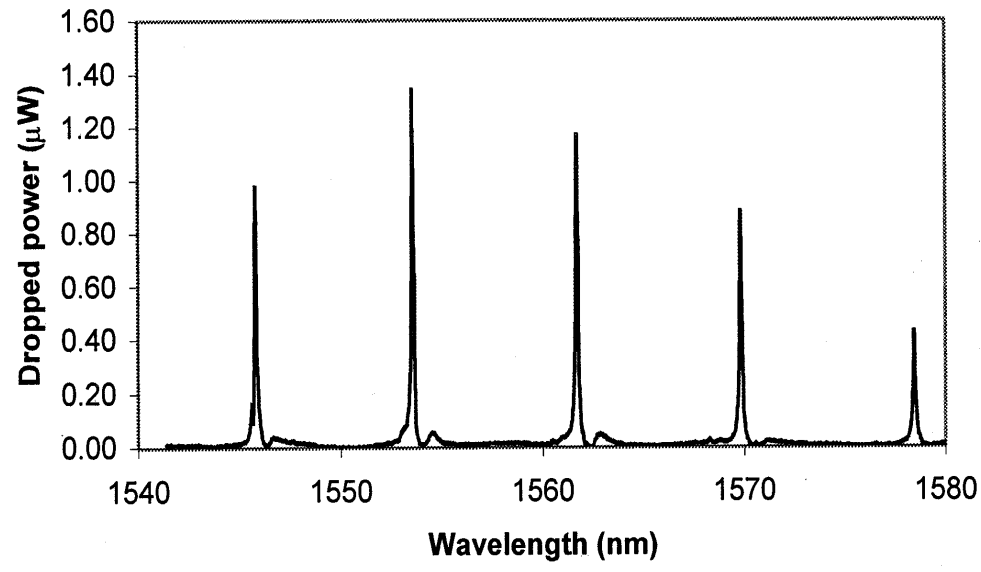

(a)

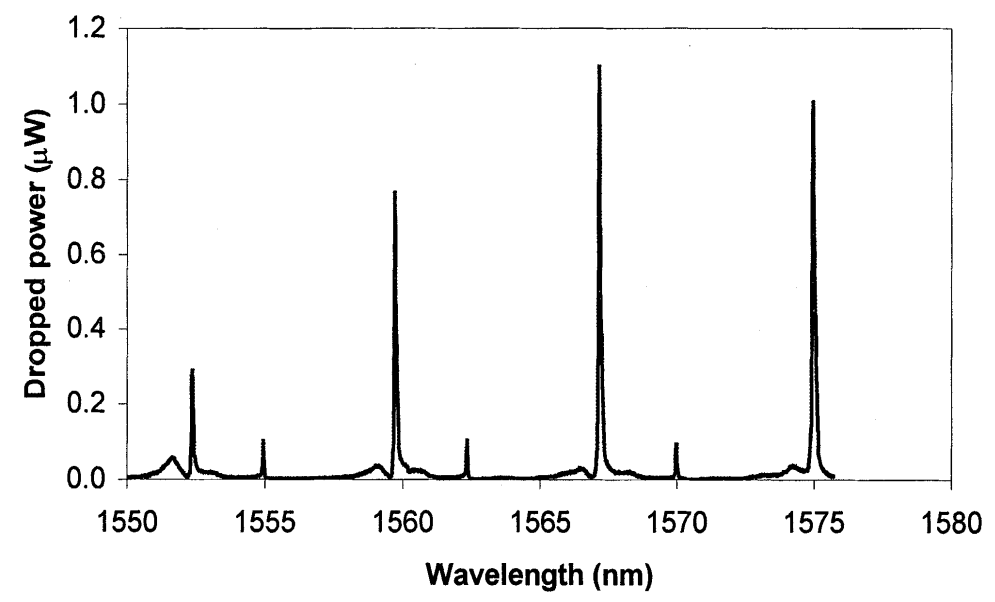

(b)

Figure 2: Drop port power as a function of wavelength of vertically coupled micro ring resonators. (a) 55 $\mu \mathrm{m}$ diameter and (b) $64 \mu \mathrm{m}$ diameter. Higher order modes can be observed in (b).

The replica molding process is shown in Fig. 3. UV curable polymer, which can be the same SU-8 (Micro-Chem Corp.) as the master patterned by e-beam, can be used because PDMS is transparent to UV light. The precision of the gap is critical to the device performance, and soft lithography truthfully replicates the quarter micron gap. Recently, soft lithography has been demonstrated to be an excellent tool to make micro-ring resonators of the same high optical performance as those made by e-beam lithography ${ }^{7}$, as shown in Fig. 4. 


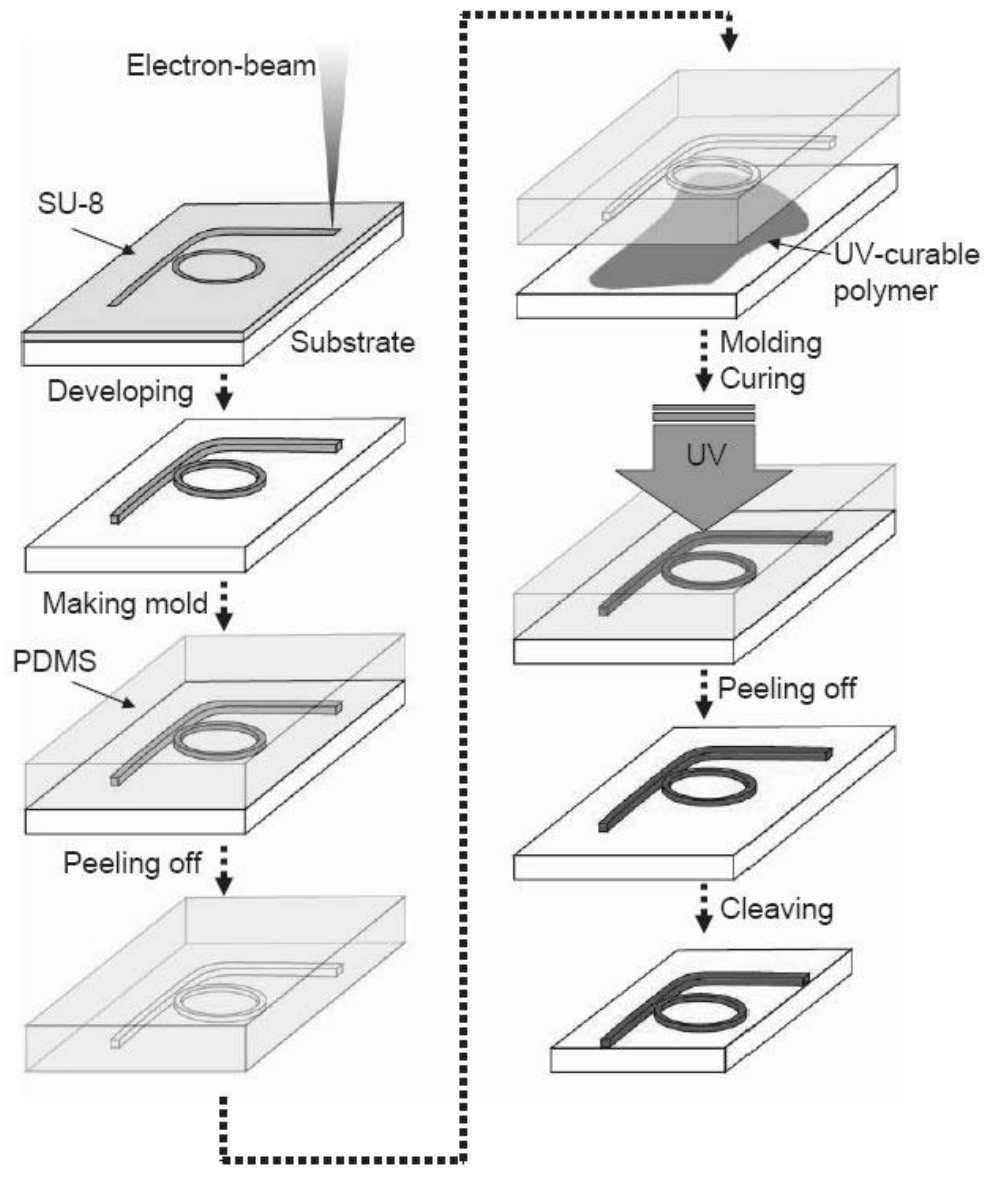

Figure 3: Soft lithography process steps. The device in the experiment is made with SU-8 polymer. The waveguide cross section is 2 um by $2 \mathrm{um}$, and the gap between the ring and the bus is $0.25 \mathrm{um}$.

\subsection{Polymer micro-ring resonators made by two-photon polymerization on side-polished single mode optical} fiber $^{10}$

A ring resonator device must be fiber attached for it to have practical uses. The total fiber-to-fiber insertion loss is always an important factor of device performance. Most of the insertion loss of a waveguide micro-ring resonator comes from the coupling loss between the optical fiber and waveguides, as well as the propagation loss of the bus waveguides. Using optical fiber as the bus waveguides can completely eliminate these sources of losses. The concept of micro-ring resonator on side polished fiber is to partially remove part of the cladding to gain access of the light in the core of the fiber by evanescent coupling. A polymer micro-ring is then fabricated on the polished flat on the fiber and vertically coupled to the core of the fiber. The total device will potentially have very low loss and very compact size. 

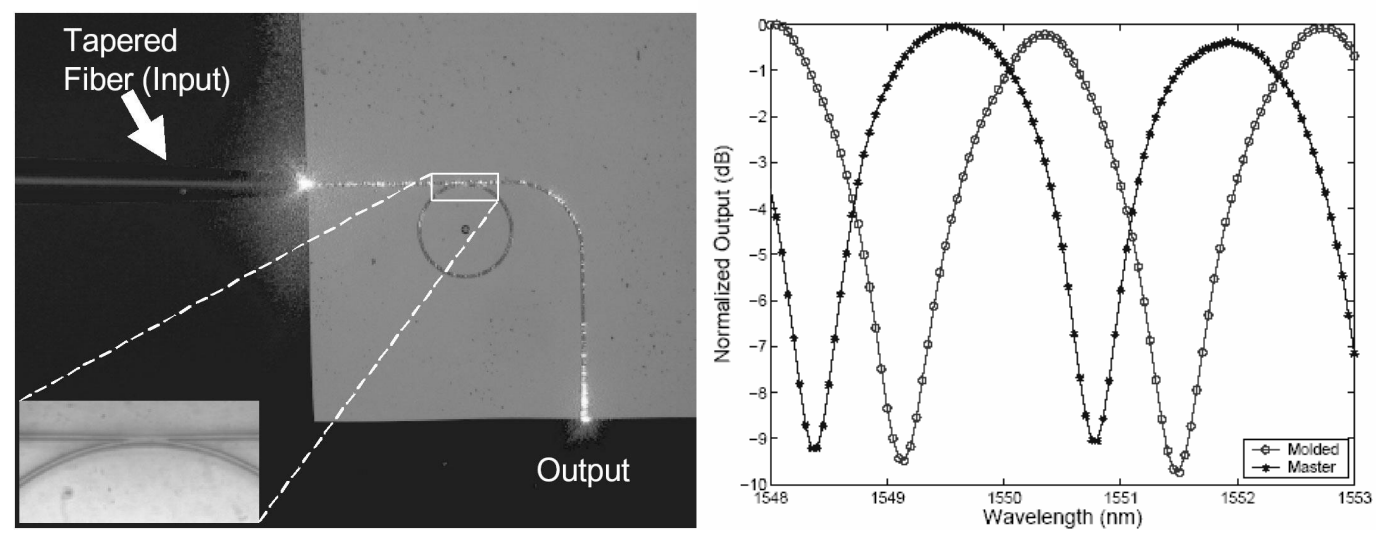

Figure 4: Polymer micro-ring resonator made my soft lithography micro molding (Left). Comparison between the transmission spectra of master micro-ring resonator optical filter and molded one (Right).

To demonstrate this novel concept, a polished flat is made on a piece of standard single mode fiber (Corning SMF28). The insertion loss and polarization dependent loss (PDL) were monitored during the polishing to determine the polishing endpoint. The ring waveguide was made by two-photon polymerization process, in which two near infrared photons from a femtosecond laser are absorbed simultaneously by non-linear optic chromophores in the monomer solution to emit a ultraviolet photon, which polymerizes the monomers ${ }^{11}$. Two-photon polymerization has a sharp threshold and polymerization only takes place within a small volume in the center of the focal point. A resolution of $200 \mathrm{~nm}$ or better can be routinely achieved. By scanning the focal point in the monomer, 3-D micro-structures of any design can be sculptured. Fig. 5 is a microscope picture of a ring resonator on side polished fiber, and Fig. 6 shows the shifting of the resonances as different pressures were applied on the ring. Shifting of resonances was also observed when varying temperature. The rings fabricated by two-photon polymerization exhibit a high level of mechanical robustness. The rings can be dislodged and repositioned using micro manipulator tips, as shown in Fig.7. This provides a very convenient way to experiment with various ring positions and degree of coupling. Even micro-rings standing vertically on the fiber are possible, as evidenced in Fig. 8.
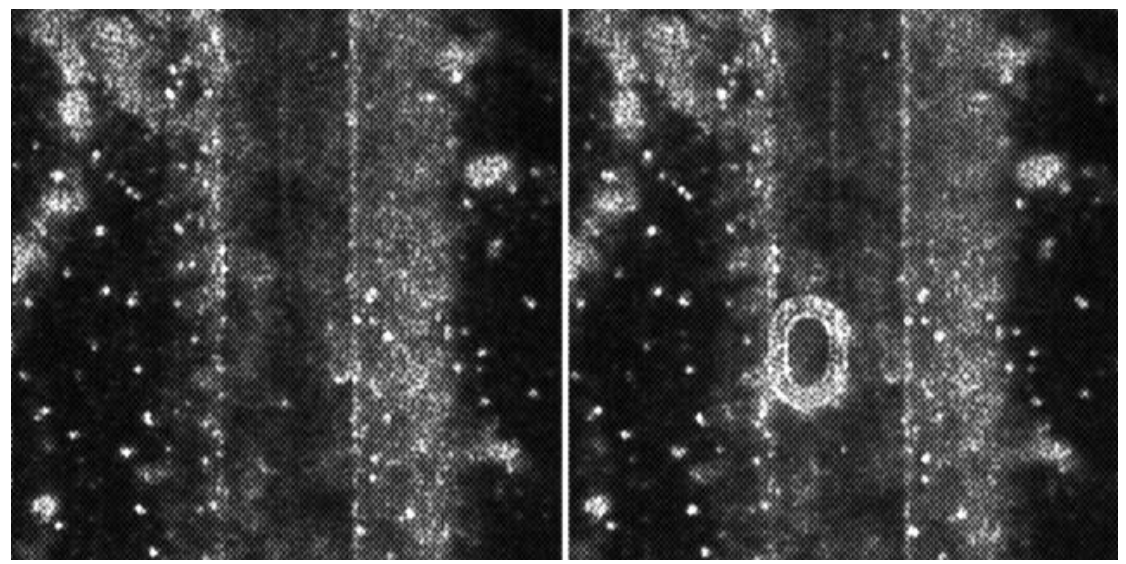

Figure 5: An oval-shaped micro-ring before and after coupled to the fiber core. 


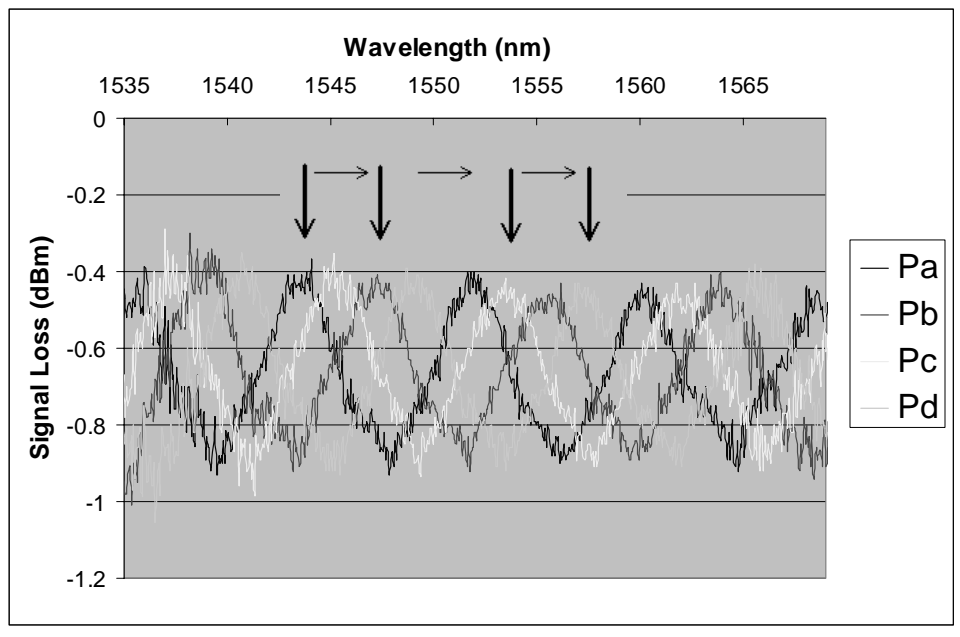

Figure 6: Resonance shift with increasing pressure.

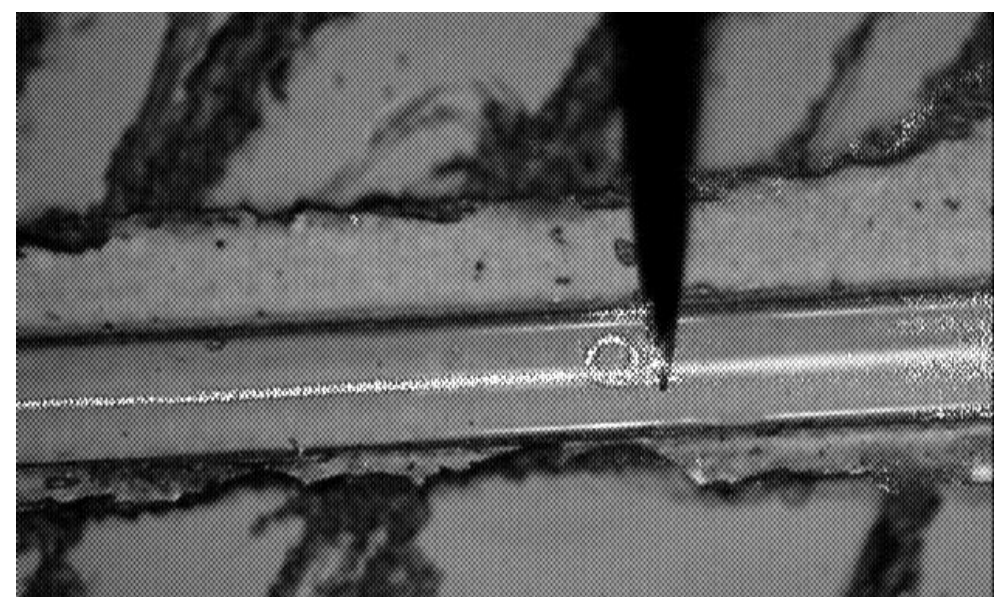

Figure 7: Ring waveguide manipulated by the microprobe to align with the core.

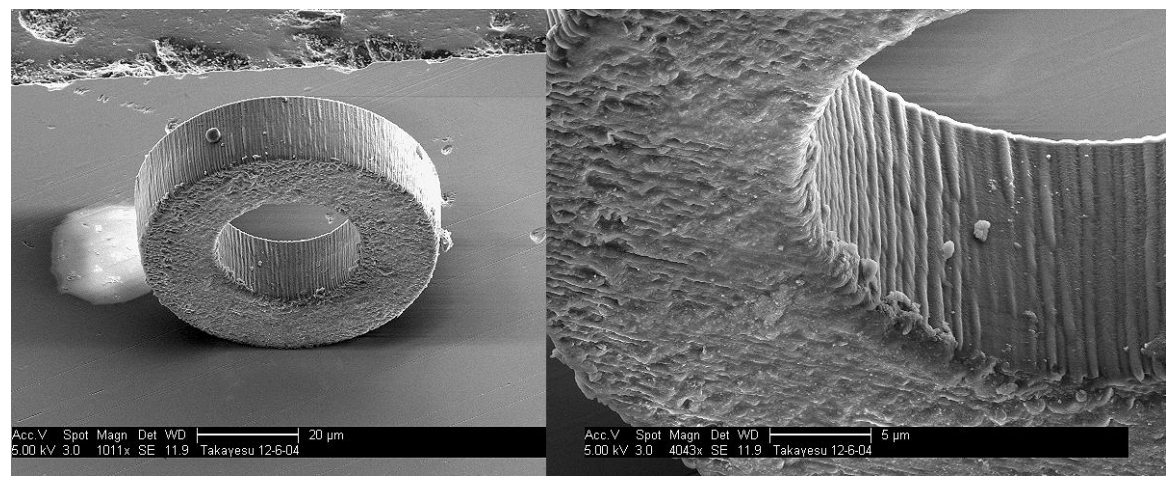

Figure 8: SEM photography of a vertically oriented ring waveguide on a side-polished optical fiber. 


\section{DYNAMIC AND PERMANENT ADJUSTMENTS OF RING RESONATORS}

Many applications such as tunable add/drop filters, signal switching, and drift compensation require that the resonant wavelengths of ring resonators be able to be dynamically changed. The resonant wavelength is determined by the round-trip optical path length of the ring waveguide. The optical path length is the product of the effective index of refraction of the ring waveguide and the physical circumference of the ring. The resonance can be effectively changed by changing the index of refraction of the ring waveguide or the cladding. On the other hand, permanent adjustment (trimming) of the ring resonators is useful to compensate for the fabrication errors and improve the fabrication yield and device performance.

\subsection{Dynamic tuning}

\subsubsection{Thermal tuning ${ }^{8}$}

The indices of refraction of polymers change with temperature. This can be used effectively to thermally tune the resonant wavelength over a large range, as shown in Fig.9.

Temperature Tuning of SU-8

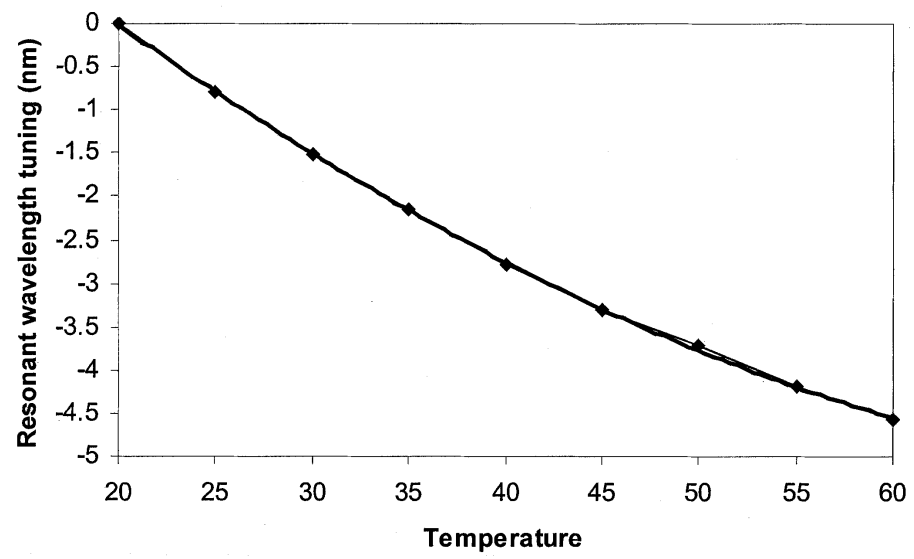

Figure 9: The thermal tuning of the $55 \mu \mathrm{m}$ diameter microresonator @ $1550 \mathrm{~nm}$. The structure of the device is shown in Fig. 1.

\subsubsection{Electro-optical tuning}

Thermal tuning can achieve a large tuning range. However, it is relatively slow and the tuning heater consumes certain amount of power. For applications that require faster tuning and/or low power consumption, electro-optic tuning provides a good solution. Electro-optic polymers change their index of refraction when subjected to an external electric field. They have demonstrated THz response speed ${ }^{12}$. Only a static voltage is needed for tuning. The power consumption is very low because the device does not draw current. With a $750 \mu \mathrm{m}$ diameter resonator made with CLD1/APC electro-optic polymer has show a tuning rate of $0.82 \mathrm{GHz} / \mathrm{V}$ at the wavelength of $1330 \mathrm{~nm}^{8}$.

\subsubsection{Liquid crystal cladding tuning ${ }^{13}$}

Large free-space range requires micro-rings of small diameter. To make small rings with acceptable bending loss, high index contrast between the ring waveguide and its claddings is needed. High index contrast is available with silicon-on-insulator (SOI) technology, in which the core of the optical waveguide is silicon $(n=3.3)$. Low loss and high-Q ring resonators have been reported with radii down to a few microns ${ }^{14,15}$. Tuning of SOI miro-ring resonators 
can be realized by using liquid crystal as the cladding material. By orienting the liquid crystal directors with an external electrostatic field, large change of refractive index can be achieved and utilized to tune the resonances.

A $5 \mu \mathrm{m}$ radius silicon waveguide ring resonator is built on silicon-on-insulator substrate. The waveguide is $205 \mathrm{~nm}$ thick by $500 \mathrm{~nm}$ wide, on $1 \mu \mathrm{m}$ oxide. A pair of electrodes was made on the opposite sides of the ring resonator. A nematic liquid crystal (Merck E63, $\mathrm{n}_{\mathrm{o}}=1.517$ and $\mathrm{n}_{\mathrm{e}}=1.744$ ) is then spin coated on the waveguides as cladding. Upon applying a DC voltage on the electrodes, the liquid crystal molecules in the cladding aligns parallel to the electrical field force lines, changing the index of refraction and resonant wavelengths, as shown in Fig.10. A tuning rate of -0.01 $\mathrm{nm} / \mathrm{V}$ at the wavelength of $1530 \mathrm{~nm}$ was observed.

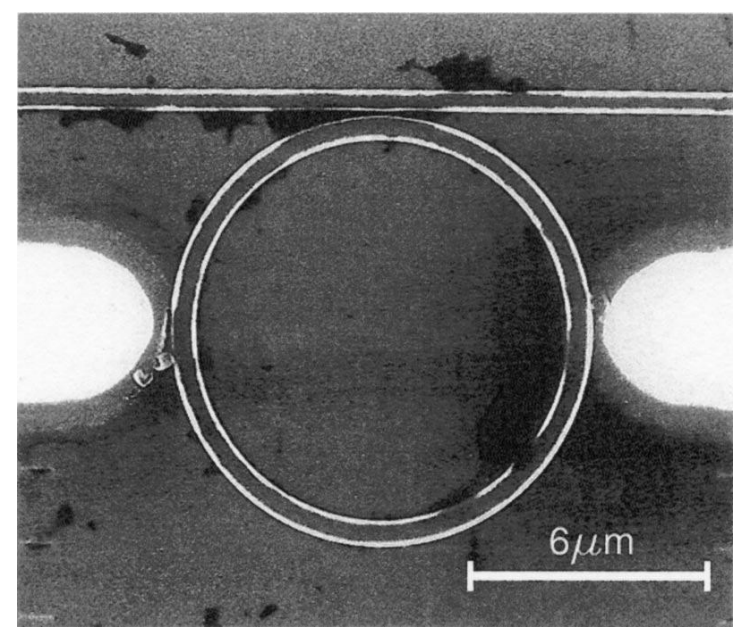

Figure 10: A scanning electron microscopy image of the ring resonator with modulation electrodes.

\subsection{Permanent trimming ${ }^{17}$}

When EO polymer is exposed to high intensity light of wavelength shorter than its absorption peak wavelength, the index of refraction of the polymer can be permanently lowered through photobleaching. This property of chromophore containing polymers have been previously used for trimming Y-branch of Mach-Zehnder modulators and directional coupler switches ${ }^{16}$. Based on this principle, a large range trimming of polymer ring resonators was successfully demonstrated.

The trimming is done by focusing a broadband visible light on a portion of the ring. The shift of resonances is clearly shown in Fig.11. The resonant wavelength can be tuned by as much as $8 \mathrm{~nm}$. After photobleaching, the color of the polymer becomes clear from its original color of green, indicating a permanent photo-decomposition of chromphores in the electro-optic polymer.

Permanent trimming is useful to compensate for the device fabrication variations. Devices after trimming can still be dynamically tuned by temperature or electrical field.

\section{DEVICES MADE OF POLYMER MICRO-RING RESONATORS}

\subsection{EO polymer micro-ring modulators ${ }^{8}$}

By tuning the resonance in and out of the fixed wavelength of a signal channel, switching and modulation can be achieved. Fig.12 is a micro-ring resonator made with CLD1/APC electro-optical polymer waveguides. A ground electrode is fabricated under the waveguide layer stack and a top electrode is fabricated on top of the upper cladding 
directly above the resonator ring. The intensity of the light at the output port is modulated by the voltage on the electrode (Fig.13). Modulation at $1 \mathrm{~Gb} / \mathrm{s}$ has been demonstrated with a simple lumped electrode design. High modulation speed is expected with traveling wave electrode of optimized design. Compared to conventional MachZehnder modulators of a few centimeters in length, the micro-ring modulators are only a few hundred microns in size.

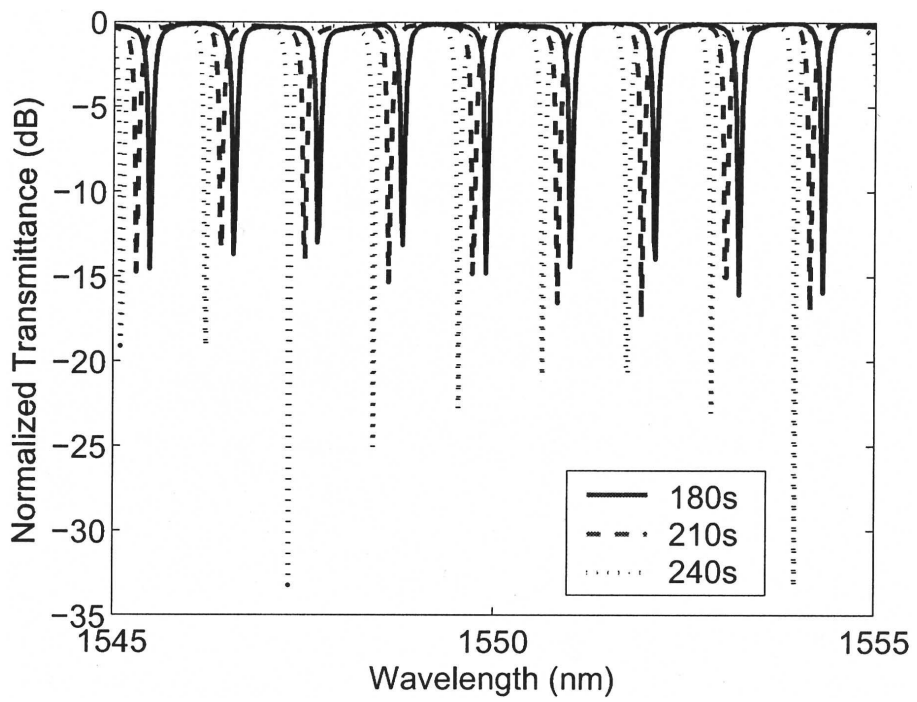

Figure 11: Tuning of the micro-ring resonances. The transmission spectra are for TE-polarized light after various exposure times. The resonances shift by approximately $-0.2 \mathrm{~nm}$ after each 30 -s exposure with a light source intensity of approximately $35 \mathrm{~mW} / \mathrm{cm}^{2}$ over a $0.28 / \mathrm{mm}^{2}$ area.
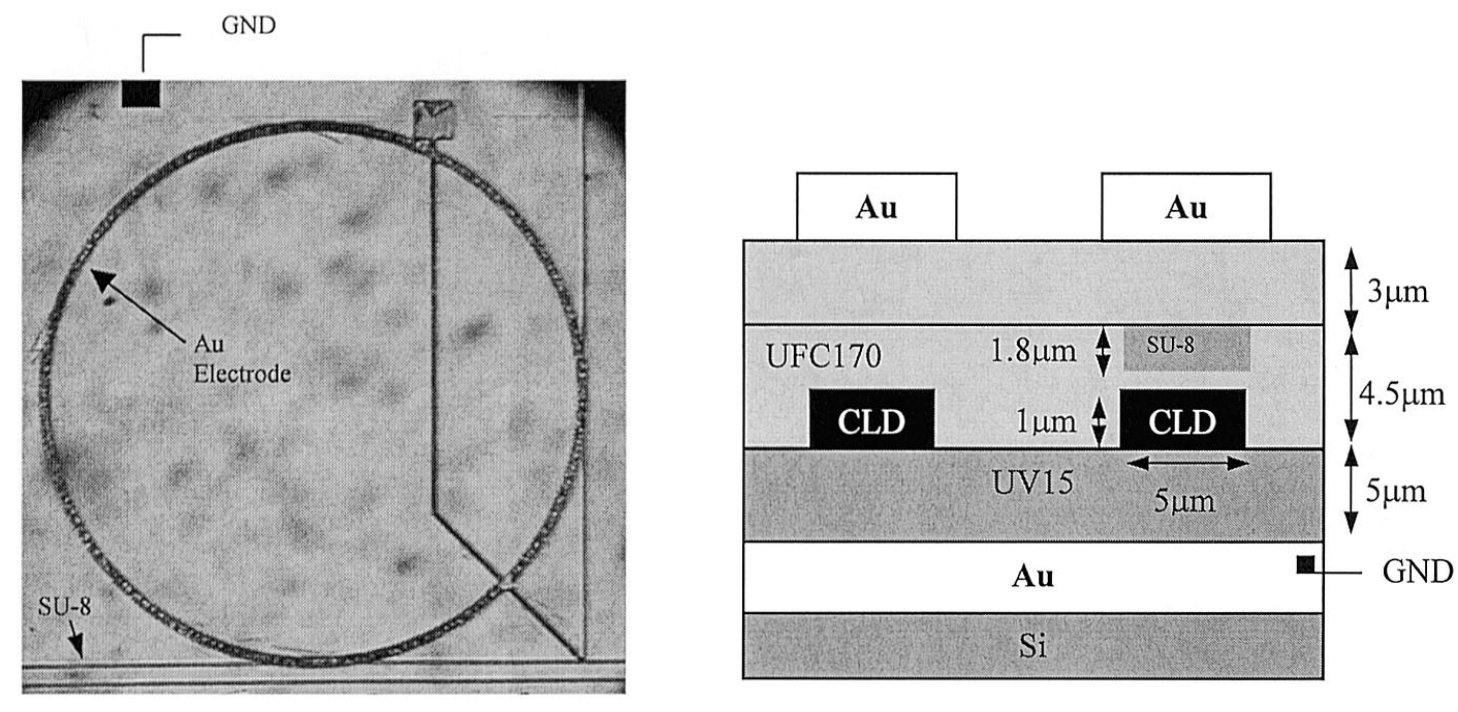

Figure 12: A microscope photography of an electro-optic micro-ring modulator (left) and its cross-section structure (right).

\subsection{Mach-Zehnder interferometer using serially coupled micro-ring resonators ${ }^{18}$}

In conventional MZ waveguide interferometers, both arms are made of channel waveguides. The amplitude of the light in each arm remains constant. Only the phases change. The variation in the phase difference between the two waveguides causes constructive and destructive interference at the output of the interferometer. A Mach-Zehnder interferometer of unequal optical path lengths for each arm acts like a wavelength filter. However, the transmission 
spectrum is sinusoidal instead of with sharp pass (or reject) bands. Replacing one channel waveguide arm with cascaded micro-ring resonators creates a novel Mach-Zehnder interferometer wavelength filter. The output from the arm consists of serially coupled ring resonators varies in both phase and amplitude as the function of wavelength. The interference between the serially coupled ring resonator arm and the channel waveguide arm produces sharp periodical maximums and minimums in the output(Fig. 14), which agrees well with a simple matrix model analysis. This novel device is interesting in nonlinear optics research, as well as optical filtering and modulation applications.

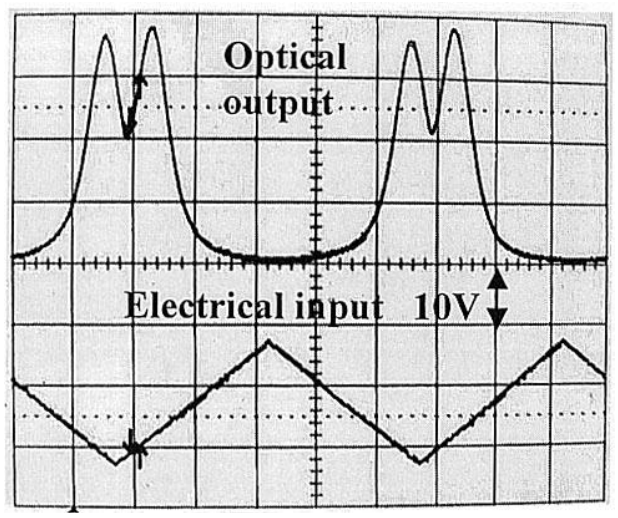

Figure 13: The applied voltage to the electrodes of the device and the modulated output intensity at $1300 \mathrm{~nm}$ for a 1500 $\mu \mathrm{m}$ diameter device at a fixed wavelength.

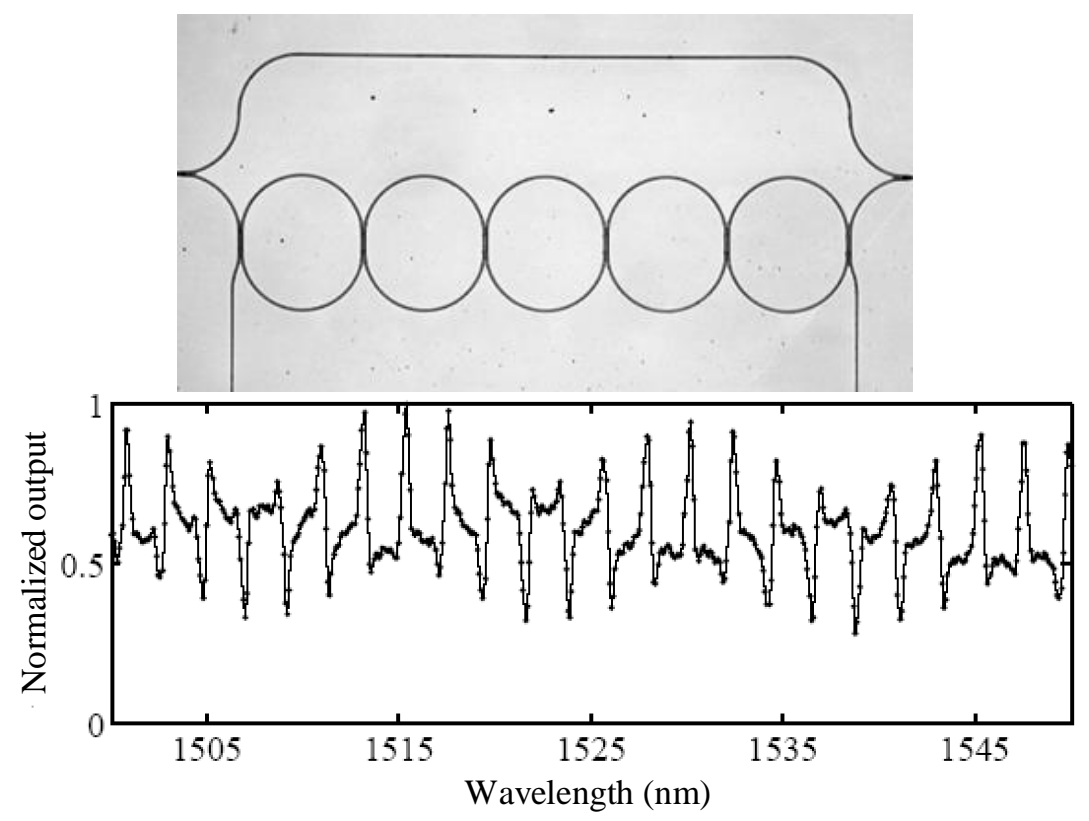

Figure 14: Mach-Zehnder interferometer with serially coupled micro-rings and its transmission spectrum. The device is built on silicon substrate with $5 \mu \mathrm{m}$ oxide layer. The waveguide is made with SU-8 and is $1.2 \mu \mathrm{m}$ thick and $2 \mu \mathrm{m}$ wide, the coupling gap is $750 \mathrm{~nm}$. The rings have $50 \mu \mathrm{m}$ straight coupling sections and $100 \mu \mathrm{m}$ bend radii in the curved sections. The total length of the device is $1.2 \mathrm{~mm}$. 


\section{CONCLUSIONS}

Taking advantage of the process flexibility of polymeric materials, micro-ring resonators can be fabricated with several technologies, including conventional photolithography and etching, soft lithography micro molding, and twophoton polymerization. These micro-ring resonators can be tuned thermally and/or electro-optically. They can also be permanently trimmed after fabrication. Wavelength filters, add/drop multiplexers/demultiplexers, and electro-optical modulators have been demonstrated using passive and electro-optic polymers. Unlike inorganic materials with fixed physical properties, organic polymers can be designed and synthesized to have desired properties. As the advancement in passive and electro-optic polymers continues, polymer micro-ring resonator devices of higher performance and new functions will be possible.

\section{REFERENCES}

1. M. K. Chin, C. Youtsey, W. Zhao, T. Pierson, Z. Ren, S. L. Wu, L. Wang, Y. G. Zhao, and S. T. Ho, IEEE Photonics Technol. Lett., 11, 1620 (1999).

2. B. E. Little, S. T. Chu, H. A. Haus, J. Foresi, and J.-P. Laine, J. Lightwave Technol., 15, 998 (1997).

3. S. Suzuki, Y. Hatakeyama, Y. Kokubun, and S. T. Chu, J. Lightwave Technol., 20, 745 (2002).

4. $\quad$ K. Oda, N. Takato, and H. Toba, J. Lightwave Technol., 9, 728 (1991).

5. S. T. Chu, B. E. Little, W. Pan, T. Kaneko, S. Sato, and Y. Kokubun, IEEE Photonics Technol. Lett., 11, 691 (1999).

6. $\quad$ L.J. Guo, C-Y. Chao, W. Fung, and J. Yang, App. Phys. Lett., 83, 1527 (2003).

7. Y. Huang, G. T. Paloczi, J. Scheuer, and A. Yariv, Optics Express, 11, 2452 (2003). http://www.opticsexpress.org/abstract.cfm?URI=OPEX-11-20-2452

8. $\quad$ P. Rabiei, W.H. Steier, Z. Cheng, and L.R. Dalton, "Polymer micro-ring filters and modulators," $J$. Lightwave Technol., 20, 1968 (2002).

9. Y. Xia and G.M. Whitesides, Annual Review of Material Science, 28, 153 (1998).

10. T. Sherwood, C. Young, J. Takyesu, A. Jen, L. Dalton, and A. Chen, Proceedings of SPIE, 5724, paper 58, in press (2005).

11. X-M. Duan, H-B. Sun, and S. Kawata, Journal of Photopolymer Sci. and Tech., 17, 393 (2004).

12. M. Lee, H.E. Katz, C. Erben, D.M. Gill, P. Gopalan, J.D. Heber, and D.J. McGee, Science, 298, 1401 (2002).

13. B. Maune, R. Lawson, C. Gunn, A. Scherer, and L. Dalton, Applied Phys. Lett., 83, 4689 (2003).

14. D. Rafizadeh, J. P. Hagness, A. Taflove, K. A. Stair, and S. T. Ho, Opt. Lett., 22, 1244 (1997).

15. D. Rafizadeh, J. P. Zhang, R. C. Tiberio, and S. T. Ho, J. Lightwave Technol., 16, 1308 (1998).

16. A. T. Chen, V. Chuyanov, F. I. MartiCarrera, S. Garner, W. H. Steier, S. S. H. Mao, Y. S. Ra, L. R. Dalton, and Y. Q. Shi, IEEE Photon. Technol. Lett., 9, 1499 (1997).

17. J. K. S. Poon, Y. Huang, G. T. Paloczi, and A. Yariv, C. Zhang, and L.R. Dalton, Opt. Lett., 29, 2584 (2004).

18. George T. Paloczi, Yanyi Huang, and Amnon Yariv, and Shayan Mookherjea, Optical Express, 11, 2666 (2003). 